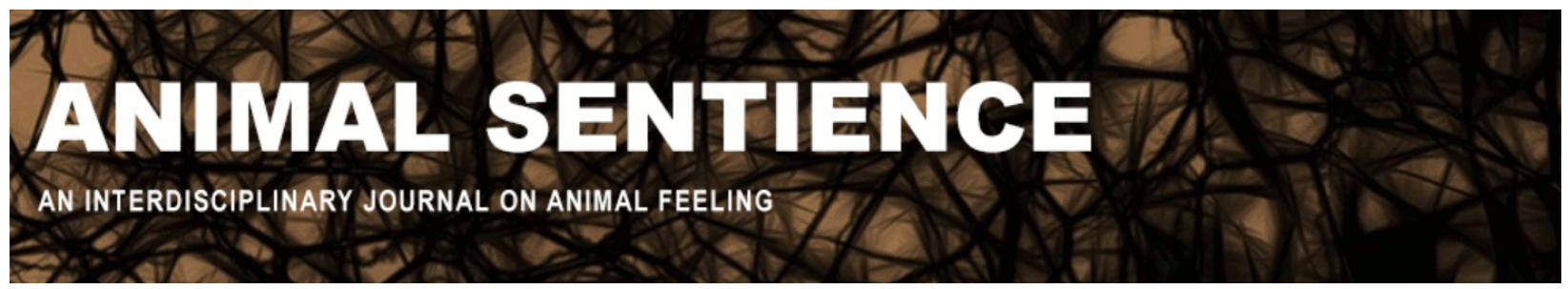

Harmon-Jones, Eddie and Harmon-Jones, Sylvia K. (2018) On jealousy, envy, sex differences and temperament in humans and dogs. Animal Sentience 22(8) DOI: $10.51291 / 2377-7478.1338$

Date of submission: 2018-05-28 Date of acceptance: 2018-05-31 (c) 


\title{
On jealousy, envy, sex differences and temperament in humans and dogs
}

Commentary on Cook et al. on Dog Jealousy

\author{
Eddie Harmon-Jones \& Sylvia K. Harmon-Jones \\ The University of New South Wales
}

\begin{abstract}
Cook, Prichard, Spivak, and Berns (2018) find that dogs' levels of trait aggression are positively correlated with their amygdala activation when observing their caregivers giving a food to a fake dog. The authors conclude that this may provide neural evidence in dogs for the experience of jealousy, an emotion that some psychologists consider to be unique to humans. Here we explain the difference between the emotions of jealousy and envy, suggesting some ideas for future experiments that may help disentangle the experience of jealousy from that of envy in dogs. We also propose ideas for future research that may yield a more in-depth understanding of jealousy, and whether jealousy exists, in non-human animals.
\end{abstract}

Eddie Harmon-Jones, Professor of Psychology at The University of New South Wales, studies the effects of emotions on attention and other cognitive processes, the role of emotion and motivation in aggressive and pro-social behaviour, as well as the antecedents and consequences of discrepancies between cognitions. Website

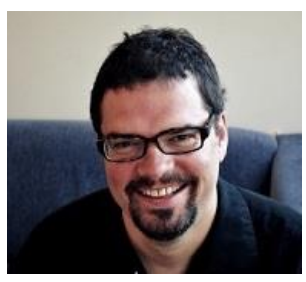

Sylvia K. Harmon-Jones is an Honours student in Psychology at The University of New South Wales. She is currently examining early behavioral predictors of anxiety and associated biomarkers.

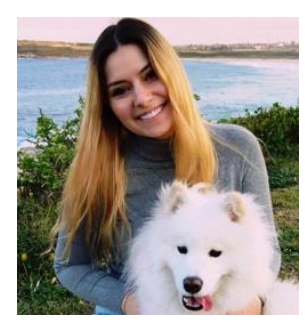

Cook, Prichard, Spivak, and Berns (2018) have conducted an interesting study examining the neural responses of 13 domestic dogs during three events: (1) the dog receiving food from his caregiver; (2) a fake dog receiving food from the real dog's caregiver; and (3) food being deposited in a bucket by the caregiver. Each dog's aggressive temperament was also assessed. The results revealed that dogs with more aggressive temperaments had heightened amygdala activation when the fake dog received food from the caregiver than when the caregiver deposited food into the bucket (condition 1 was simply used to keep the dog in the scanner and attentive to food). Cook et al. suggest that these results "may have some similarity to human jealousy."

Below we offer our thoughts on this research, which we find impressive both conceptually and for its difficult methodology. We hope our comments are taken in the spirit they are offered: thoughts for designing future research to test interesting and important research questions about jealousy in dogs.

What Psychological Response Was Evoked in the Dogs? Is the observed response more due to envy rather than jealousy? Envy is often "directed at another or others, wanting their 
qualities, success, or possession. Jealousy involves thinking you will lose, or have lost, some affection or security from another person because of someone or something else - including their interest in an activity that takes time away from you" (Lamia, 2013). We wonder whether affection or attention given to another dog from the caregiver would result in a response more similar to jealousy as observed in humans.

Most dog-owners report that their dog experiences jealousy - and primarily when the owner interacts with a third party (Morris, Doe, \& Godsell, 2008). The most commonly reported third party is another human, followed by a dog or another animal. Dogs may recognize that the other human poses more of a threat to the loss of their relationship than the other dog does. A future experiment could compare affection shared between a fake dog and the caregiver with affection between the caregiver and another human. This may suggest that dogs have an awareness that humans are more attached to other humans than they are to dogs, or that dogs prefer human contact to contact from other dogs.

Thoughts about the Experimental Design. Cook et al.'s results are correlational. No evidence is presented that would allow us to know whether the "jealous" condition evoked more amygdala activation than comparison conditions. With the three conditions included in the design, it is understandable why these tests could not be performed. The design needs a "neutral" condition to compare to the psychologically interesting conditions. The condition in which the dog is fed could not serve this purpose, because this condition is not neutral.

The authors seem to think that they could not improve their design to eliminate the alternative explanation that aggressive dogs experience increased amygdala activation in the presence of another dog. That is, the dogs may not have experienced jealousy when the fakedog received a treat; rather they may have simply experienced arousal as their attention was brought to the fake dog. However, the caretaker could give the fake dog an unpleasant-tasting piece of food (color-coded so that the dog knows that it is unpleasant). This should bring attention to the fake dog without eliciting jealousy/envy.

Comparing a machine (or another human unimportant to the dog) giving food to the fake dog with the caregiver giving the food would also be interesting. This would assist in disentangling whether the increased amygdala activity was due to contact between the caregiver and the fake dog (indicating jealousy), or due to envy/reward inequality. But inclusion of more than three conditions within one experiment may be impossible given the amount of time that dogs can remain relatively motionless and attentive for fMRI scanning.

What Does Amygdala Activation Indicate Psychologically? The amygdala is a complex neural region that can be divided into 13 nuclei and cortical areas (in nonhuman primates), each with distinct psychological and behavioral processes (Freese \& Amaral, 2009). At present, fMRI research is unable to localize activity to these individual areas. Moreover, $\mathrm{fMRI}$ research with adult humans has revealed that the amygdala response is associated with many psychological processes, including both positive and negative feelings as well as ambivalence (Cunningham \& Brosch, 2012).

Cook et al. recognize that the amygdala is involved in a variety of psychological processes and in the end conclude that their research demonstrates that the dogs exposed to the "jealousy" induction were more aroused (rather than that they experienced some more specific psychological state). If the research is only aimed at measuring arousal, then peripheral physiological measures could be used. These measures would be less expensive than $\mathrm{FMRI}$ and would not suffer the limitations associated with preventing the dogs from 
moving. For instance, measurement of impedance cardiography together with respiration could distinguish sympathetic and parasympathetic nervous system contributions to cardiac activity. Sympathetic nervous system activity is associated with the psychological construct of arousal.

Other Neural Activations? With this rich fMRI dataset, it is surprising that the researchers did not test for activation in other neural regions. Several other neural regions may be related to jealousy. For example, in humans, insula activity is associated with bodily responses in emotions (Craig, 2011); the anterior cingulate cortex is associated with psychological pain related to social rejection (Eisenberger, Lieberman, \& Williams, 2003) (if they experienced jealousy, then dogs should have experienced this pain); and the medial prefrontal cortex is involved in social processing (Mitchell, Macrae, \& Banaji, 2005).

Past research has revealed that jealousy is associated with relatively greater activation in the left than the right dorsolateral frontal cortical region in humans (Harmon-Jones, Peterson, \& Harris, 2009). More recent research using transcranial direct current stimulation (tDCS) to increase activity in the left frontal cortical region has revealed that this region plays a causal role in evoking jealousy (Kelley, Eastwick, Harmon-Jones, \& Schmeichel, 2015). Thus, a comparison of left versus right frontal activity may also indicate whether dogs' neural patterns suggest that they experience jealousy akin to that experienced by humans, as well as whether jealousy in dogs is associated with approach motivation.

Sex Differences and Temperaments. As Cook et al. note, some research suggests that men and women differ in which neural regions are activated during jealousy (Takahasi et al., 2006). Cook et al. did not indicate the sex of the dogs used in their research. Inclusion of a larger sample with equal numbers of male and female dogs would permit testing for sex differences in dogs' neural responses to jealousy-evoking events. Such research would add immensely to the human research on sex differences in jealousy; for example, dogs may not have been exposed to as much of the alleged social influence on sex as humans.

The C-BARQ scale (used for aggressiveness) measures 14 traits. Examining the correlation between some of these other traits and neural activations to jealousy would be interesting. In particular, Separation-Related Problems and Attachment/Attention Seeking might relate to more intense jealousy-related responses, or activation in different brain regions associated with jealousy. As mentioned by Cook et al., jealousy is a blend emotion, and thus trait aggression may not be the primary correlate of jealousy in dogs. Non-aggressive dogs may also experience intense feelings of jealousy that are associated with activation in other brain regions. The most frequent behavior demonstrated by dogs experiencing jealousy, according to dog-owners, is pushing against the owner or between the owner and the other person/animal (Morris, Doe, \& Godsell, 2008), suggesting that dogs can express jealousy without being aggressive. 


\section{References}

Cook, P., Prichard, A., Spivak, M., \& Berns, G. S. (2018). Jealousy in dogs? Evidence from brain imaging. Animal Sentience 22(1).

Craig, A. D. (2011). Significance of the insula for the evolution of human awareness of feelings from the body. Annals of the New York Academy of Sciences, 1225(1), 72-82.

Cunningham, W. A., \& Brosch, T. (2012). Motivational salience: Amygdala tuning from traits, needs, values, and goals. Current Directions in Psychological Science, 21(1), 54-59.

Eisenberger, N. I., Lieberman, M. D., \& Williams, K. D. (2003). Does rejection hurt? An fMRI study of social exclusion. Science, 302(5643), 290-292.

Freese, J. L., \& Amaral, D. G. (2009). Neuroanatomy of the primate amygdala. In P. J. Whalen \& E. A. Phelps (Eds.), The human amygdala (pp. 3-42). New York, NY: Guilford Press.

Harmon-Jones, E., Peterson, C. K., \& Harris, C. R. (2009). Jealousy: Novel methods and neural correlates. Emotion, 9, 113-117.

Kelley, N. J., Eastwick, P. W., Harmon-Jones, E., \& Schmeichel, B. J. (2015). Jealousy increased by induced relative left frontal cortical activity. Emotion, 15, 550-555.

Lamia, M. C. (2013). Jealousy and envy: The emotions of comparison and contrast. Psychology Today.

Mitchell, J. P., Macrae, C. N., \& Banaji, M. R. (2005). Forming impressions of people versus inanimate objects: Social-cognitive processing in the medial prefrontal cortex. Neuroimage, 26(1), 251-257.

Morris, P. H., Doe, C., \& Godsell, E. (2008). Secondary emotions in non-primate species? Behavioural reports and subjective claims by animal owners. Cognition and Emotion, 22(1), 3-20.

Takahashi, H., Matsuura, M., Yahata, N., Koeda, M., Suhara, T., \& Okubo, Y. (2006). Men and women show distinct brain activations during imagery of sexual and emotional infidelity. Neurolmage, 32(3), 1299-1307. 


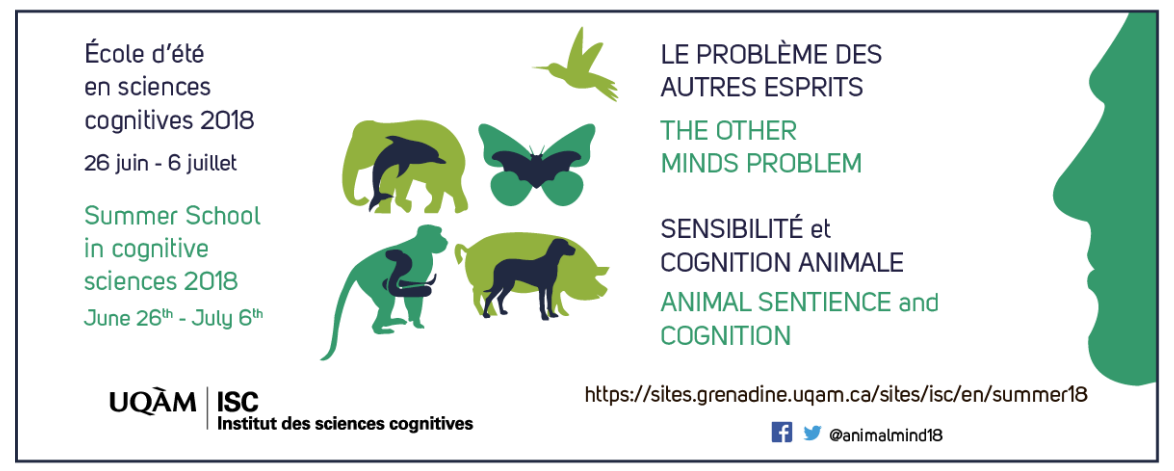

ISC 2018 Summer School in Cognitive Sciences June 26 - July 6, 2018 Montreal (Canada) The Other Minds
Problem: Animal
Sentience and Cognition

Overview. Since Descartes, philosophers know there is no way to know for sure what — or whether — others feel (not even if they tell you). Science, however, is not about certainty but about probability and evidence. The 7.5 billion individual members of the human species can tell us what they are feeling. But there are 9 million other species on the planet (20 quintillion individuals), from elephants to jellyfish, with which humans share biological and cognitive ancestry, but not one other species can speak: Which of them can feel — and what do they feel? Their human spokespersons - the comparative psychologists, ethologists, evolutionists, and cognitive neurobiologists who are the world's leading experts in "mindreading" other species - will provide a sweeping panorama of what it feels like to be an elephant, ape, whale, cow, pig, dog, chicken, bat, fish, lizard, lobster, snail: This growing body of facts about nonhuman sentience has profound implications not only for our understanding of human cognition, but for our treatment of other sentient species.

|Gregory Berns: Decoding the Dog's Mind with Awake Neuroimaging

Gordon Burghardt: Probing the Umwelt of Reptiles

Jon Sakata: Audience Effects on Communication Signals

PANEL 1: Reptiles, Birds and Mammals

WORKSHOP 1: Kristin Andrews: The "Other" Problems: Mind,

Behavior, and Agency

Sarah Brosnan: How Do Primates Feel About Their Social

Partners?

Alexander Ophir: The Cognitive Ecology of Monogamy

Michael Hendricks: Integrating Action and Perception in a Small

Nervous System

PANEL 2: Primates, Voles and Worms

WORKSHOP 2: Jonathan Birch: Animal Sentience and the

Precautionary Principle

Malcolm Maclver: How Sentience Changed After Fish Invaded

Land 385 Million Years Ago

Sarah Woolley: Neural Mechanisms of Preference in Female

Songbird

Simon Reader: Animal Social Learning: Implications for

Understanding Others

PANEL 3: Sea to Land to Air

WORKSHOP 3: Steven M. Wise: Nonhuman Personhood

Tomoko Ohyama: Action Selection in a Small Brain (Drosophila

Maggot)

Mike Ryan: "Crazy Love": Nonlinearity and Irrationality in Mate

Choice

Louis Lefebvre: Animal Innovation: From Ecology to

Neurotransmitters

PANEL 4: Maggots, Frogs and Birds: Flexibility Evolving

SPECIAL EVENT: Mario Cyr: Polar Bears

Colin Chapman: Why Do We Want to Think People Are

Different?

Vladimir Pradosudov: Chickadee Spatial Cognition

Jonathan Balcombe: The Sentient World of Fishes

PANEL 5: Similarities and Differences

WORKSHOP 5 (part 1): Gary Comstock: A Cow's Concept of

Her Future

WORKSHOP 5 (part 2): Jean-Jacques Kona-Boun: Physical

and Mental Risks to Cattle and Horses in Rodeos
Joshua Plotnik: Thoughtful Trunks: Application of Elephant Cognition for Elephant Conservation

Lori Marino: Who Are Dolphins?

Larry Young: The Neurobiology of Social Bonding, Empathy and Social Loss in Monogamous Voles

Panel 6: Mammals All, Great and Small

WORKSHOP 6: Lori Marino: The Inconvenient Truth About

Thinking Chickens

Andrew Adamatzky: Slime Mould: Cognition Through

Computation

Frantisek Baluska \& Stefano Mancuso: What a Plant Knows and Perceives

Arthur Reber: $\mathrm{A}$ Novel Theory of the Origin of Mind:

Conversations With a Caterpillar and a Bacterium

PANEL 7: Microbes, Molds and Plants

WORKSHOP 7: Suzanne Held \& Michael Mendl: Pig Cognition and Why It Matters

James Simmons: What Is It Like To Be A Bat?

Debbie Kelly: Spatial Cognition in Food-Storing

Steve Phelps: Social Cognition Across Species

PANEL 8: Social Space

WORKSHOP 8: To be announced

Lars Chittka: The Mind of the Bee

Reuven Dukas: Insect Emotions: Mechanisms and Evolutionary Biology

Adam Shriver: Do Human Lesion Studies Tell Us the Cortex is

Required for Pain Experiences?

PANEL 9: The Invertebrate Mind

WORKSHOP 9: Delcianna Winders: Nonhuman Animals in

Sport and Entertainment

Carel ten Cate: Avian Capacity for Categorization and

Abstraction

Jennifer Mather: Do Squid Have a Sense of Self?

Steve Chang: Neurobiology of Monkeys Thinking About Other Monkeys

PANEL 10: Others in Mind

WORKSHOP 10: The Legal Status of Sentient Nonhuman

Species 